\title{
An Educational Platform in Structural Mechanics
}

\author{
http://dx.doi.org/10.3991/ijoe.v9iS8.3319 \\ Tiago A. N. Silva ${ }^{1,2}$ and Maria A. R. Loja ${ }^{1,2}$ \\ ${ }^{1}$ ADEM/ISEL - Instituto Superior de Engenharia de Lisboa, \\ GI-MOSM - Grupo de Investigação em Modelação e Optimização de Sistemas Multifuncionais, \\ Polytechnic Institute of Lisbon, Portugal. \\ 2 IDMEC/LAETA - Instituto de Engenharia Mecânica, \\ University of Lisbon, Portugal.
}

\begin{abstract}
Thermal residual stresses often arise due to a manufacturing process, involving localised thermal induction, or to the existence of structural components with different thermal expansion coefficients. The existence of thermal residual stresses within a structural member is usually undesired, as it decreases the mechanical resistance of structures. Hence, it is desirable to obtain both a minimum level of residual stresses and smoother stresses transitions in the materials interfaces. Regarding the mitigation of thermal residual stress concentration, the use of materials which properties can vary along the component directions has great interest. This work addresses the use of dual-phase functionally graded materials, which microstructure varies gradually from a material to another according to a given gradation function. On the order hand, it is also addressed the use of a population based optimization algorithm in order to attain the referred minimum stress level. Summarizing, the current work presents an educational platform directed to structural mechanics students, which aims to give the tools to understand both the influence of design parameters in the thermal residual stress level and distribution along the material and the advantages of using a structural optimization technique in order to minimize the drawback thermal residual stresses effects.
\end{abstract}

Index Terms-Educational platform; Virtual experiments; Mechanical engineering; Graded materials.

\section{INTRODUCTION}

Several usual industrial material processing and manufacturing processes involve localized plastic deformation or thermal induction, such as in the case of structures welding or composite laminates curing, where localized addition or residual energy, in conjunction with the use of components made of different constituents, leads to the growth of thermal residual stresses. Since functionally graded materials (FGM) appeared, they are known to provide superior thermal and mechanical performances when compared to the traditional laminated

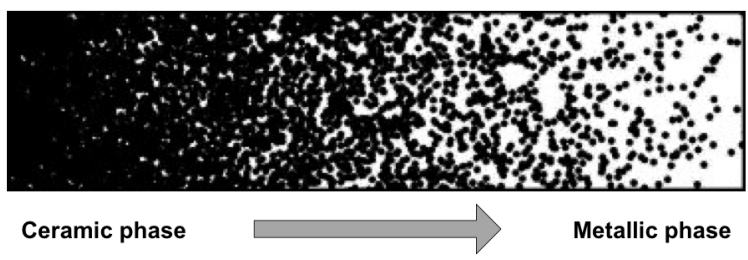

Figure 1. Illustration of a functionally graded material (adapted from [1]). composites [2]. Among the FGM properties characteristics, the continuous variation of its material constituents enables smoother stress distribution profiles due to the avoidance of abrupt material transitions, and consequently, of residual stress concentrations. In addition, the mechanical behaviour of a structure can be improved if structural optimization is addressed. Hence, besides the opportunity to test and analyse several material and structural configurations, the authors also consider the use of the differential evolution (DE) algorithm [3] in order to attain an optimized structure, regarding the minimization of the thermal residual stress level [4].

Students in the mechanical engineering M.Sc. level at ISEL already have acquired knowledge in areas such as composite materials and mechanics of deformable bodies. Therefore, the present work is intended to give a holistic view of mechanical engineering, where each student can be motivated to work not confined to a specific course curricular content [5]. Hence, being aware of the capabilities of virtual experiments to reach and to encompass students in educational or research activities, the current work intends to present an educational platform built in LabVIEW $^{\circledR}$ [6] and integrated with MATLAB $^{\circledR}$ [7] in order to study the phenomena of thermal residual stresses in functionally graded structures

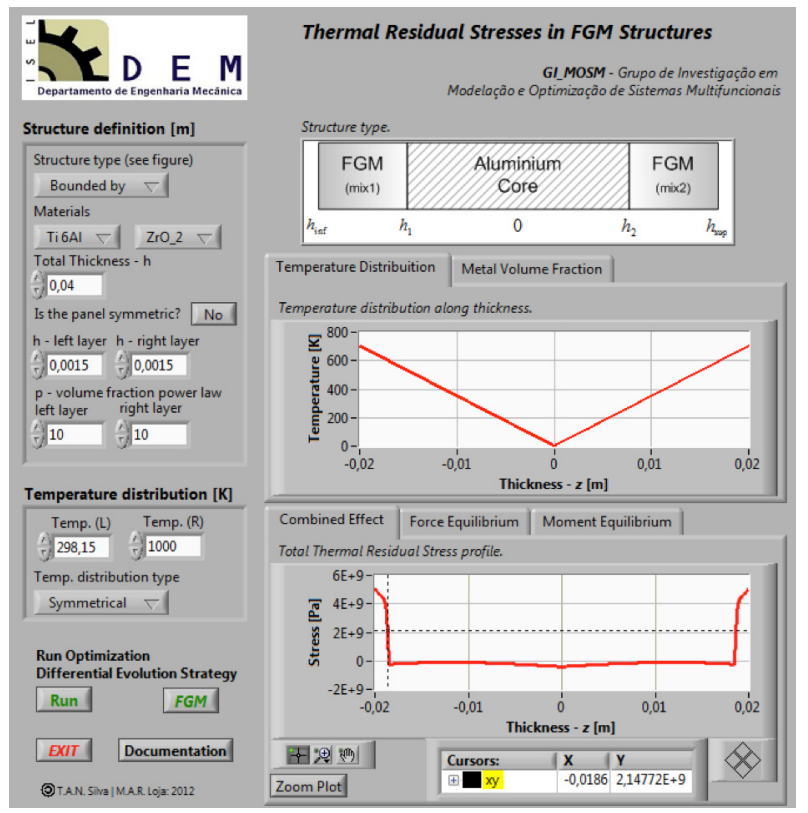

Figure 2. Main frontend of the educational platform. 
and its minimization regarding structural optimization.

\section{A FunCtionally GRAdEd SANDWiCH StRUCTURE}

Regarding composite materials, one can find materials which constituents are spherical or nearly spherical particles embedded in an isotropic matrix in such a way that the overall material properties vary continuously along a given material direction, the FGM. In this work, it is addressed the use of a dual-phase FGM constituted by metallic and ceramic particles (Fig. 1). Also in typical fibre reinforced laminated composites exists the concept of sandwich structures. Sandwich structures can be defined as a layer wise combination of materials, and therefore, it allows exploiting the advantages of FGM without a significant weight addition, as it is usual to use ceramic materials. Frequently FGM structures are used as thermal barriers and consequently subjected to high temperature environments, being necessary to take into account the effective material properties, which is a function of the temperature [8]. Thus, to predict the effective mechanical behaviour of a sandwich panel which core is metallic and its outer layers are made of a FGM, one considers the Voigt rule of mixtures [9] with the volume fraction of metal across the thickness given by a power law [8], e.g. Fig. 1 gives an example of a linear volume fraction of metal.

\section{Thermal RESIDUAL STRESSES}

If a material or structure is submitted to a manufacturing process or similar that involves cooling down the structure after a high temperature processing, often thermal residual stresses arise. Among the most common causes of thermal residual stresses is the localised heating due to weldment. Regardless the motive, the fact is that the difference in the thermal expansion characteristics of a structure's constituents is an inevitable drawback. However, the use of FGM structures can adjust the thermal residual stresses magnitude and distribution, not only in order to achieve smoother material transitions but also to generate compressive residual stresses, which increase the mechanical resistance of structures subjected to bending loads.

In the present work, the authors addressed the study of three main structural configurations: a sandwich panel

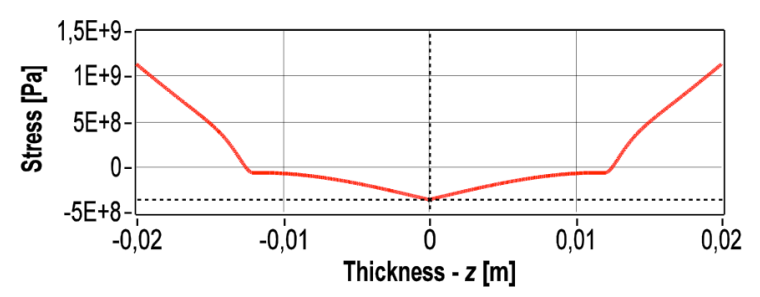

a) Titanium alloy and silicon nitride.

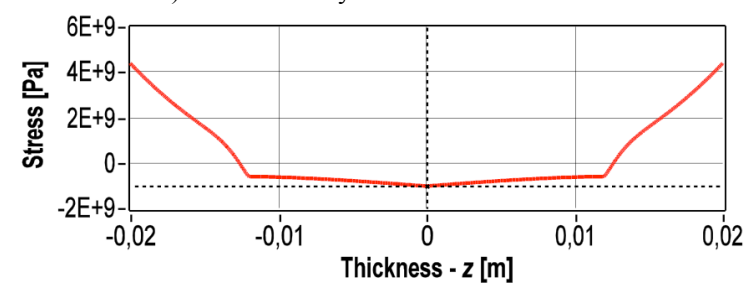

b) Titanium alloy and zirconia.

Figure 3. Thermal residual stress distribution, for different FGM constituents, considering a symmetrical temperature distribution.

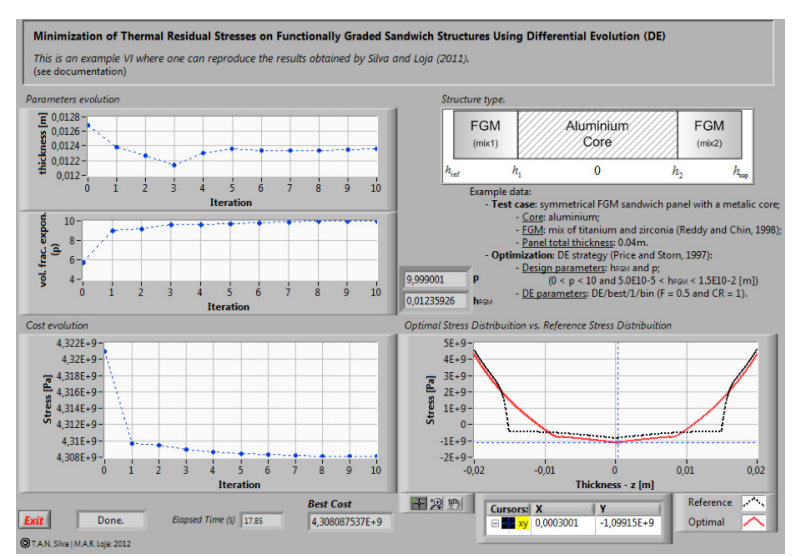

Figure 4. Frontend dedicated to the optimal design of a given FGM sandwich structure [4].

with both outer layers made of FGM; a laminated panel with just one FGM layer; and a single FGM panel. Note that the panel is considered to be infinitely long in the width direction, and the thickness characteristics can be tuned by the platform user (Fig. 2). Further details on the definition of thermal residual stresses developed in kind of structures can be found in [10].

Fig. 3 depicts the thermal residual stress distribution obtained for a symmetrical sandwich panel with both outer layers made of two different FGMs. Note that if different FGM constituents are considered different thermal residual stress distribution and magnitude can be obtained, for the same temperature distribution, and therefore students are enabled to understand the effect of choosing different materials on the thermal residual stress values.

\section{StRUCTURAL Optimization}

Regarding the optimization context, despite the technique or approach, it is usual to deal with either a minimization or a maximization problem. As referred, in terms of thermal residual stresses, it is desirable to minimize the stress level, through the estimation of a set

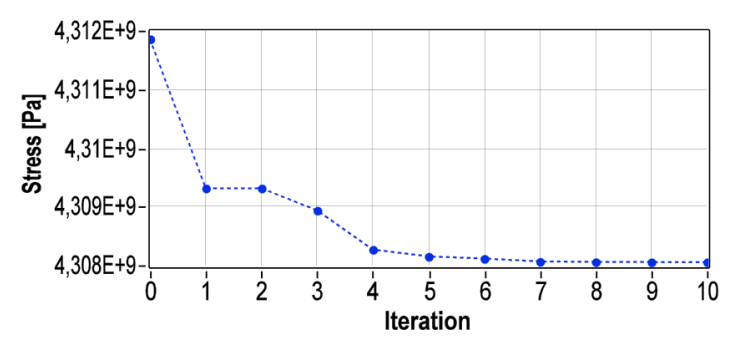

Figure 5. Evolution of the objective function value during the optimization process.

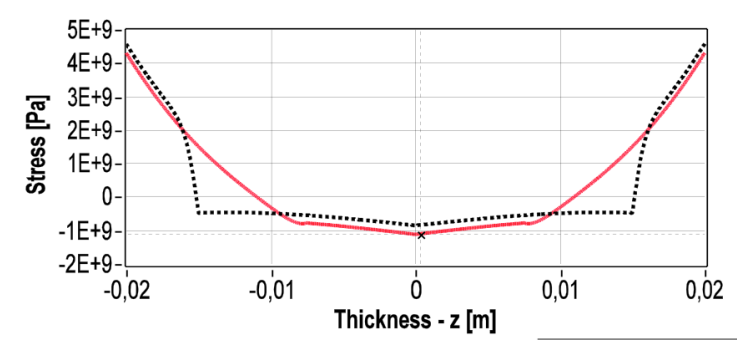

Figure 6. Thermal residual stress distribution, before (dashed line) and after (solid line) optimization. 
of model parameters or design variables.

$\mathrm{DE}$ is a population-based approach to objective function optimization that generates trial individuals by computing the difference between randomly selected members of the population. Generically, given an objective function to be minimized, the DE algorithm begins by randomly generating population vectors per design variable. The generated vectors or individuals will evolve over the optimization procedure, simulating an evolving population. Hence, for each generation of individuals, the objective function is evaluated several times in order to find the best population member, meeting a predefined criterion. DE congregates characteristics from global optimization as well as search direction vectors, which preserves an important descent property.

The educational platform under discussion, besides the presented capabilities, has a frontend entirely dedicated to a structural optimization application example (Fig. 4), where students can track the evolution of the optimization process (Fig. 5) and evaluate both the optimal solution (Fig. 6) and its corresponding set of design variable.

\section{The Educational Platform - Overview}

With a didactic purpose, the authors present an educational platform developed through the integrated use of MATLAB ${ }^{\circledR}$ and LabVIEW ${ }^{\circledR}$. As case study, the authors exploit the thermal residual stress phenomenon developed in sandwich structures with metallic core and dual-phase FGM layers, different structural configurations and materials selection are available. By the selection of several structural related design parameters, the user of the proposed platform acquire some knowledge about the impact of different temperature distributions, symmetry consideration and materials combinations on the magnitude and distribution of thermal residual stresses. Note that the use of this tool is thought to be directed to students at the M.Sc. degree in mechanical engineering, as the approached subjects are somehow an extension of the already acquired knowledge both on structural mechanics and optimization. Moreover, due to its graphical capabilities, the presented educational tool is intended to motivate and to enhance students' competences on the referred field of knowledge.

The present educational platform is divided into four main applications, concerning different focuses [11]. Hence, the user is invited to explore, separately, the influence of several design parameters in the magnitude and distribution of thermal residual stresses and the behaviour of the DE algorithm; and to assess the application of $\mathrm{DE}$ on structural optimization via the minimization of the magnitude of thermal residual stresses in a FGM sandwich panel. Moreover, there is available a documentation area where the user can find reference works and general information on the treated matters.

\section{CONCLUDING REMARKS}

This paper gives an overview of the phenomenon of thermal residual stresses developed in functionally graded materials and briefly presents the differential evolution global optimization technique. However the main focus of the present work is an educational platform that enables students to exploit the effect of the adequate design parameters on the referred subjects. This educational tool aims to promote students' motivation and competences in mechanical design and structural optimization. This platform is built in LabVIEW $^{\circledR}$, due to its graphical and web-publishing capabilities, although all calculus related code is reused from already available MATLAB ${ }^{\circledR}$ codes. Note that this is not due to limitations of LabVIEW ${ }^{\circledR}$ but an option in order to stimulate scholar to deliver more appellative working tools to students without the need of recoding or translating every single application to a software with enhanced graphical capabilities.

\section{ACKNOWLEDGMENT}

This work was partially supported by the Portuguese Foundation for Science and Technology (FCT) through the Project PTDC/ATP-AQI/5355/2012 and the PhD grant SFRH/BD/44696/2008.

\section{REFERENCES}

[1] F. J. Ferrante, S. R. Arwade, and L. L. Graham-Brady, "A translation model for non-stationary, non-Gaussian random processes," Probabilis Eng. Mech., vol. 20, no. 3, pp. 215-228, July 2005. http://dx.doi.org/10.1016/j.probengmech.2005.05.003

[2] M. Koizumi, "The concept of FGM," Ceram. Trans., vol. 34, pp. 3-10, 1993.

[3] R. Storn and K. Price, "Differential evolution - a simple and efficient heuristic for global optimization over continuous spaces," J. Global Optim., vol. 11, pp. 341-359, 1997. http://dx.doi.org/10.1023/A:1008202821328

[4] T. A. N. Silva and M. A. R. Loja, "Differential Evolution on the Minimization of Thermal Residual Stresses in Functionally Graded Structures," in Computational Intelligence and Decision Making Trends and Applications, From Intelligent Systems, Control and Automation, vol. 61, A. Madureira, C. Reis, and V. Marques, Eds. Netherlands: Springer, 2013, pp. 289-299.

[5] X. Du, E. de Graaff, and A. Kolmos, Research on PBL Practice in Engineering Education, Rotterdam: Sense Publishers, 2009.

[6] National Instruments Co., LabVIEW 2009.

[7] The MathWorks, Inc. MATLAB R2009b.

[8] J. N. Reddy and C. D. Chin, "Thermo-mechanical analysis of functionally graded cylinders and plates," J. Therm. Stresses, vol. 21, no. 6, pp. 593-626, 1998. http://dx.doi.org/10.1080/014957 $\underline{39808956165}$

[9] L. X. Li and T. J. Wang, "A unified approach to predict overall properties of composite materials," Mater. Charact., vol. 54, no. 1, pp. 49-62, January 2005. http://dx.doi.org/10.1016/j.matchar. 2004.10.005

[10] K. S. Ravichandran, "Thermal residual stresses in a functionally graded material system," Mat. Sci. Eng. A-Struct., vol. 201, no. 12, pp. 269 - 276, October 1995. http://dx.doi.org/10.1016/09215093(95)09773-2

[11] T. A. N. Silva and M. A. R. Loja, "A remote virtual experiment in mechanical engineering," in $2^{\text {nd }}$ Experiment@ International Conference, 18 - 20 September 2013 in Coimbra, Portugal.

\section{AUTHORS}

T. A. N. Silva is with the Department of Mechanical Engineering, ISEL - Instituto Superior de Engenharia de Lisboa, Rua Conselheiro Emídio Navarro, 1, 1959-007 Lisboa, Portugal (e-mail: tasilva@dem.isel.ipl.pt).

M. A. R. Loja is with the Department of Mechanical Engineering, ISEL - Instituto Superior de Engenharia de Lisboa, Rua Conselheiro Emídio Navarro, 1, 1959-007 Lisboa, Portugal (e-mail: amelialoja@dem.isel.ipl.pt).

This article is an extended and modified version of a paper presented at the International Conference exp.at'13, held 18-20 September 2013, in Coimbra, Portugal. Submitted 18 November 2013. Published as resubmitted by the authors 04 December 2013. 